\title{
PROCESSING OF HIGH-SPEED CAMERA IMAGES OF HOPKINSON BAR EXPERIMENTS USING DIGITAL IMAGE CORRELATION
}

\author{
M. Adorna*, M. Neuhäuserová**, T. Fíla, , , J. Falta ${ }^{* *}$, P. Koudelka**, P. Zlámal ${ }^{* *}$
}

\begin{abstract}
In this paper, digital image correlation method (DIC) is introduced as a tool for evaluation of high strain-rate experiments perfomed using Hopkinson Bar apparatus. Samples of advanced cellular materials with predefined periodic structure and negative Poisson's ratio (auxetic structures) were investigated in this study. In-house Hopkinson Pressure Bar apparatus was used to perform the impact experiments and the experimental setup was observed using a pair of high-speed cameras. Custom DIC software tool was used to evaluate highspeed cameras records. Selected representative results of DIC applications on Hopkinson Bar experiments are provided in this paper.
\end{abstract}

Keywords: Digital Image Correlation, Hopkinson Bar, impact testing, auxetic structures, high-speed imaging

\section{Introduction}

In the recent years there has been a notable increase in terms of implementation of advanced material structures into industrial applications. For instance, modern construction solutions of deformation zones of objects subjected to extreme ways of loading (e. g. high rate impacts or blasts) require utilization of complex material structures providing high absorption of impact energy while reducing the overall weight of the resulting product. Various types of hybrid foams or materials with predefined complex structures such as auxetic structures (i. e. porous materials with negative Poisson's ratio) show a great potential in the field of energy absorption applications (e. g. safety features). It is essential to precisely describe and quantify dynamic response of such cellular structures under high strain-rate loading prior to their implementation within a complex industrial applications. Whereas Hopkinson Bar measuring technique is well established for measurements performed with conventional (e. g. bulk metallic) specimens (Wang et. al. (2015)), performing and precisely evaluating such experiments with cellular structures is much more difficult. For additional inspection, it is neccessary to observe the surface of the deformed specimen during high strainrate loading using a high speed imaging. Complex dynamic response of such materials requires introduction of not only conventional strain-gauge measuring methods but precise contactless methods such as Digital Image Correlation (DIC). DIC technique is a well established method used for assessment of full-field maps of displacement and strain on the surface of the measured complex structure. It also allows for precise determination of Poisson's ratio, which is one of the most important material characteristics of so called auxetic structures, directly from the deformation of the surface structure. Representative results of fullfield DIC application on Hopkinson Bar experiment, comparison of DIC and strain-gauge results, virtual strain-gauge method and DIC evaluation of Hopkinson Bar impact velocity are shown in this paper.

\section{Materials and methods}

\subsection{Hopkinson Bar apparatus}

Two different experimental setups based on a Hopkinson Bar apparatus are currently used for high strainrate measurements of cellular materials performed at our department. The first variant is conventional Split

\footnotetext{
Ing. Marcel Adorna: Czech Technical University in Prague, Faculty of Transportation Sciences, Konviktská 20; 110 00, Prague; CZ, adorna@fd.cvut.cz

** Czech Technical University in Prague, Faculty of Transportation Sciences, Konviktská 20; 110 00, Prague; CZ
} 
Hopkinson Pressure Bar (SHPB), a well established high strain-rate measuring device (Falta et. al. (2018)). This setup is able to reach impact velocity of $45 \mathrm{~ms}^{-1}$ with $500 \mathrm{~mm}$ aluminum striker. Thus, the setup is able to conduct experiments of the cellular structures at high strain-rates (typically $1500-4000 \mathrm{~s}^{-1}$ for structures with length in range of $12-20 \mathrm{~mm}$ ). Maximum strain in the specimen reachable in the conventional SHPB experiment is directly proportional to the striker bar length and striker bar impact velocity. Thus, for cellular structures testing very long striker bars have to be used. Moreover, maximum strain decreases with the decreasing strain-rate limiting the experimental envelope. Due to high strain-rates of the SHPB experiments, problems with achieving of dynamic equilibrium may also occur. As an alternative to the SHPB, Open Hopkison Pressure Bar (OHPB) was introduced in 2016 (see Govender and Curry (2016)), and is succesfully used for measurements of complex structures with non-homogenous deformation. OHPB is a direct impact method in which the instrumented striker bar directly hits the specimen mounted on the face of the transmission bar. Thus, this method is similar to direct impact Hopkinson Bar or e. g. "slow bar" apparatus and can be used to compress the specimen to very high strain (densification of the structure in our case). However, as the incident bar is instrumented it is possible to evaluate the data from the rear face as well as from the impact face. Dynamic equilibrium conditions can therefore be analyzed. The main disadvantages of the method are lower typical strain-rates (comparing to SHPB) and relative complexity of the system. Impact experiments are in both experimental setups observed by a pair of high speed cameras (Fastcam SA-Z, Photron, Japan) with typical frame-rate 100-300 kfps. Random speckle on the bars and on the surface of the specimens is used for analysis of the high-speed images with DIC. The Hopkinson Bar setup with two cameras and the principle of both SHPB and OHPB methods are shown Fig.1.
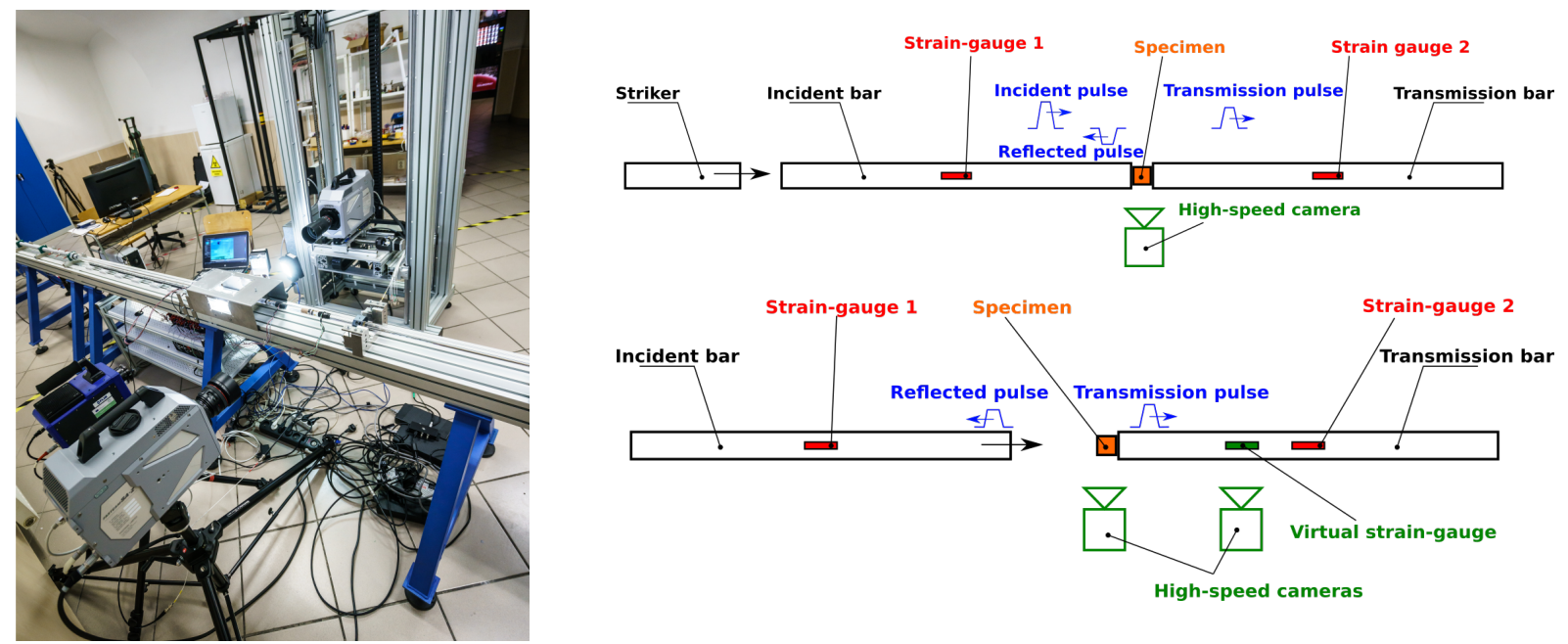

Fig. 1: Hopkinson Bar experimental setup with two high-speed cameras (left), principle of the SHPB and OHPB method with schematic depiction of high-speed cameras used in our setup (right)

\subsection{Digital Image Correlation}

Digital image correlation (DIC) is the optical method that enables to evaluate displacement and strain fileds of the object based on images of its surface. In our case this method is used for contactless measurements of displacement and strains of the specimen or of the measurement bars. The method is based on the tracking of correlation points in the series of the images taken during the experiment. Grid of virtual tracking points is defined in the first image of the undeformed surface and the changes in the locations of these points, which represents the displacement paths, are then evaluated within the whole series of the images. In order to successfully apply the DIC method, the surface of the sample has to contain a stochastic pattern, which enables to determine location of each of the tracking points based on their unique proximity using the predefined correlation criterion (Lucas and Kanade (1981)). 


\section{Applications of DIC on high strain-rate data}

\subsection{Full-field DIC analysis}

A uniform grid of $11 \times 14$ correlation points was used to cover the surface of selected 3D inverted honeycomb specimen in the images captured by high-speed camera. Custom developed evaluation DIC tool (Jandejsek et. al. (2010)) was succesfully used to track the position of the grid points and the new positions of these points were identified in the sequence of the captured high-speed camera images. Mean value of the correlation coefficient was higher than $96 \%$ up to $30 \%$ of overall strain, which illustrates reliability of the calculated results. Evolution of the full-field in-plane longitudinal strain of the auxetic structure evaluated using DIC is shown in Fig.2.

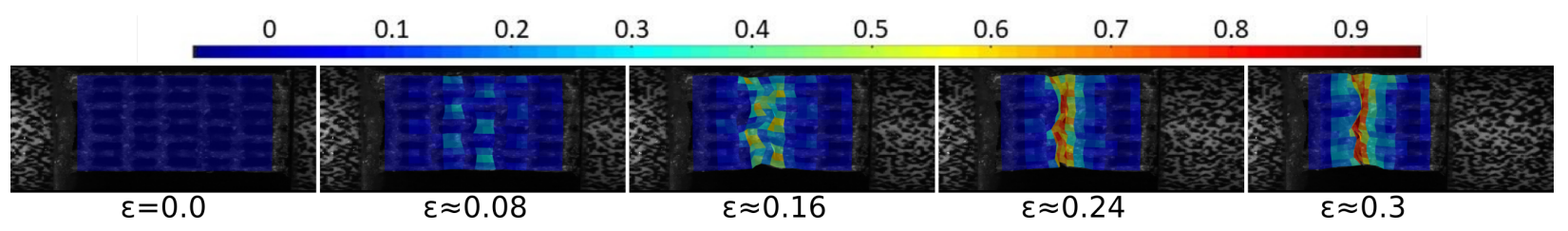

Fig. 2: Full-field DIC analysis, OHPB measurement of auxetic structure: in-plane longitudinal strain

\subsection{DIC and strain-gauge measurement results comparison}

The stress-strain curves with strain calculated using DIC and compared with the curves from conventional strain-gauge evaluation are shown in Fig. 3a. Note, that DIC strain was in this case derived by tracking grid of correlation points created not on the surface of the specimen but on the both ends of the measuring bars. Solid lines represent data evaluated from two strain-gauges which were placed on both incident and transmission bar, dotted lines represent the same curves obtained by pairing strain evaluated from DIC with stresses from the strain-gauges. Resulting curves are in a very good agreement although the experiment was not conducted in perfect dynamic equilibrium. Moreover, the rising edge in the stress-strain diagram is evaluated more precisely from the DIC than from strain-gauges that are affected by the ramp-in effect of the strain wave.

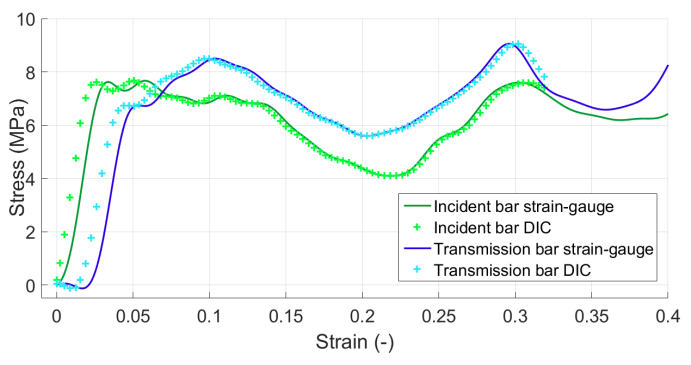

(a)

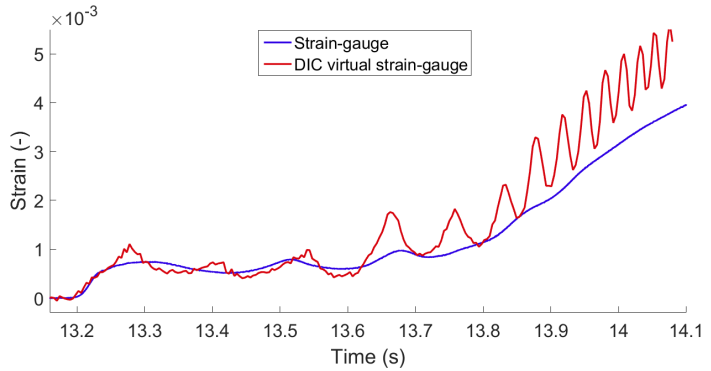

(b)

Fig. 3: (a) Comparison of stress-strain diagrams with strain evaluated from strain-gauges and DIC; (b) Comparison of strain derived from strain-gauge and DIC virtual strain-gauge

\subsection{DIC virtual strain-gauge}

DIC analysis can be used not only for the evaluation of deformation behaviour of the measured samples, but also as a tool for verification of strain-gauge elastic wave record. For this purpose, so called virtual strain gauge is created on the measuring bar. This strain-gauge consists of a small area with stochastic random pattern, which is observed by the second high speed camera. Using DIC, the strain of this virtual strain-gauge is evaluated and compared with strain curve from strain-gauge located in its close proximity (see Fig. 3b). Note, that both strain-gauge and DIC curves are in a good agreement. Scattering of the virtual strain-gauge curve in the later phase of the measurement is caused by the movement and vibrations of the measuring bar. 


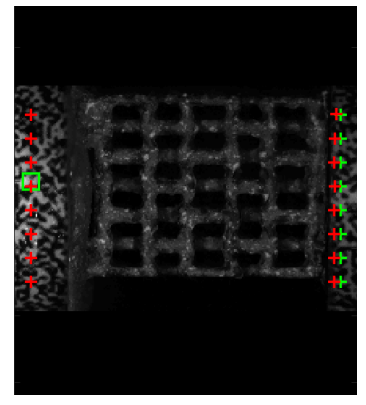

(a)

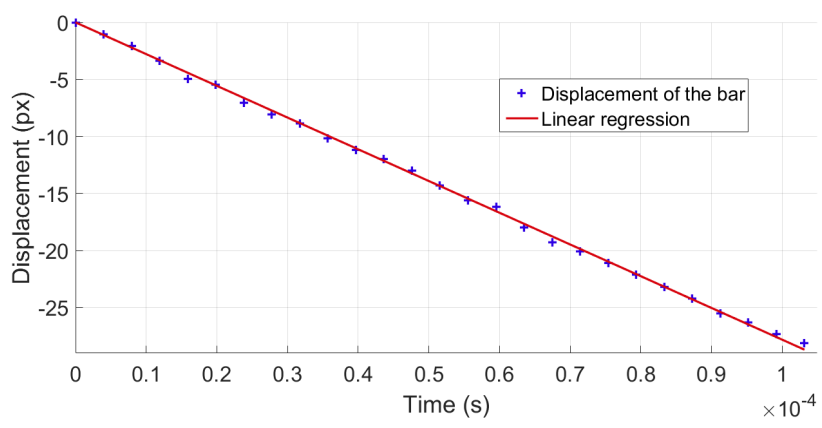

(b)

Fig. 4: (a) tracked correlation points used for evaluation of the impact velocity; (b) Displacement path of the bar prior to the impact used for the evaluation of the impact velocity using linear regression

\subsection{Hopkinson Bar impact velocity}

Impact velocity of accelerated striker is one of the input parameters of Hopkinson Bar experiment evalu ation. This parameter is particularly important in OHPB experiments as it is directly used in the calculations of strain and strain-rate in the specimen. Conventionally, impact velocity is determined using signals recorded by two through-beam photoelectric sensor (FS/FE 10-RL-PS-E4, Sensopart, Germany) installed on the barrel of the gas gun used for striker/incident bar acceleration. However, this method is not perfectly precise as the averaged value of the impact velocity is measured (lower than actual impact velocity). Using DIC it is possible to track grid of correlation points created on the both ends of the measuring bars (see Fig. 4a) and subsequently determining the real value of impact velocity using the linear regression (see Fig. $4 \mathrm{~b}$ ). For representative OHPB experiment the impact velocity determined from the through-beam sensor was $23.38 \mathrm{~ms}^{-1}$ while from DIC it reached $26.84 \mathrm{~ms}^{-1}$, which corresponds to increase in the value by ca. $15 \%$.

\section{Conclusions}

As was shown in the above-mentioned examples, the DIC is a viable method for evaluation of displacements and strains in dynamic experiments. The method enables to establish not only the full-field in-plane displacements and strains in the measured sample but also strains of virtual strain gauge placed on the measuring bar, and significantly increases the precision of the Hopkinson bar impact velocity. DIC in general is an advanced tool for analysis of deformation behaviour of materials under high strain-rate loading. However, there are still challenges which need to be faced, such as tracking of the waves on the specimen surface during non-equilibrium compression, or study of the strains during ramp-in period of the strain pulse.

\section{Acknowledgments}

The research was supported by the Czech Science Foundation (project no. 19-23675S) and the internal grants of the Czech Technical University in Prague (projects no. SGS18/153/OHK2/2T/16 and SGS18/154/OHK2/2T/16). All the financial support is gratefully acknowledged.

\section{References}

Falta, J., Fíla, T. Zlámal, P., Adorna, M. (2018) Instrumentation of Split Hopkinson Pressure Bar for Testing of Cellular Metallic Metarials, Acta Polytechnica CTU Proceedings, 18, pp 10-14

Govender R. A., Curry R. J. (2016) The “Open” Hopkinson Pressure Bar: Towards Addressing Force Equilibrium in Specimens with Non-uniform Deformation, Journal of Dynamic Behavior of Materials, 2, pp 43-49

Jandejsek I., Valach J., Vavrik D. (2010) Optimization and Calibration of Digital Image Correlation Method, Experimentalni analyza napeti 2010, pp 121-126

Lucas B. D., Kanade T. (1981) An Iterative Image Registration Technique with an Application to Stereo Vision, Proceedings of Imaging Understanding Workshop, pp 121-130

Wang P., Xu S., Li Z., Yang J., Zhang C., Zheng H. (2015) Experimental investigation on the strain-rate effect and inertia effect of closed-cell aluminum foam subjected to dynamic loading, Material Science Engineering, 620, pp 253-261 